\title{
Lung cancer surgery in octogenarians revisited-risk factors and survival in a long lifespan population
}

\author{
Wolfgang Jungraithmayr \\ Department of Thoracic Surgery, University Hospital Rostock, Rostock, Germany \\ Correspondence to: Wolfgang Jungraithmayr, MD, PhD. Department of Thoracic Surgery, University Hospital Rostock, Schillingallee 35 , 18057 \\ Rostock, Germany. Email: wolfgang.jungraithmayr@mhb-fontane.de; wolfgang.jungraithmayr@med.uni-rostock.de. \\ Provenance: This is an invited Editorial commissioned by the Section Editor Shuangjiang Li (Department of Thoracic Surgery and West China \\ Medical Center, West China Hospital, Sichuan University, Chengdu, China). \\ Comment on: Hino H, Karasaki T, Yoshida Y, et al. Risk factors for postoperative complications and long-term survival in lung cancer patients older \\ than 80 years. Eur J Cardiothorac Surg 2018;53:980-6.
}

Submitted Oct 21, 2018. Accepted for publication Dec 05, 2018.

doi: $10.21037 /$ jtd.2018.12.31

View this article at: http://dx.doi.org/10.21037/jtd.2018.12.31

The outcome of patients above 80 years undergoing lung surgery has been investigated by numerous studies proving that the majority of these patients can safely undergo effective surgery (1-3). Age itself is thus no longer a contraindication for surgery $(4,5)$. To find out which patient profits and which does not, each study identifies one or more variables that represent a potential risk factor for postoperative complications or impairs long time survival compared to their younger counterparts. For example, Berry and colleagues found that the extent of resection, use of a thoracotomy, and impaired lung function increase the risk of complications (6). However, there is no robust evidence nor is there a meta-analysis that clearly defines a particular high-risk group of patients within this population, identifying a subgroup that would profit the most from an operation, has fewest complications and the longest survival.

Thoracic surgeons have recognized the fact that the average life expectancy of octogenarians increased significantly, e.g., in Japan, the life expectancy of an 80 -year-old individual has reached approximately 9 years for men and 12 years for women. Even if we did not yet reach these age ranges in Europe and US, future medicine will provide the conditions that lead to comparable long-life expectancies in these countries. Also, there is no question that all patients, regardless of their age should receive state-of-the-art care $(4,5,7)$ given the fact that the 5 -year survival in patients with Stage I NSCLC who do not receive any form of treatment is as low as 7\% (8). Our tasks as responsible surgeons are therefore not only to operate on those patients as we would do in their younger counterparts but also to provide them post-operatively a good quality of life.

In a most recent multi-center study, Vazirani and colleagues analyzed patient data from multiple Melbourne hospitals that has the advantage of giving a more representative view of a local octogenarian population (9). Authors found that particularly patients at an age of 83 or older undergoing lobectomy are at higher risk for mortality (17\%) within a 120 -day postoperative period, and have a shorter long-term survival when compared to sub-lobar resections. Other data support the notion that elder patients within the octogenarian group perform worse than younger, e.g., age 85 and older (10), but no clear cut off value is made available so far.

Conclusively, patient selection seems to be key for a better identification of risk factors for poor outcome in elderly. Almost all publications that make an attempt to identify risk factors for elder patients employ the term "correct patient selection", but this phrase appears to be inflationary and vague. In the search for a definition of such a selection of patients, there is a nice approach by Saji and colleagues who provide for the first time a risk scoring system on 895 patients in a large observational cohort study. They identified among others male gender, Albumin $<3.8 \mathrm{ng} / \mathrm{mL}$, $\% \mathrm{VC} \leq 90$, and diabetes mellitus as predictive factors for severe postoperative complications (11). 
Others found a higher mortality in octogenarians compared to younger patients (12), speculating that the reason for this was preoperative congestive heart failure. The risk to develop congestive heart failure is a condition that can be stratified before surgery and those patients at higher risk could thus be excluded from standard operative procedures. Saji et al. furthermore reported on a higher incidence of postoperative pneumonia, respiratory failure, and supraventricular arrhythmia as well as a higher rate of cardiovascular morbidity in the octogenarian group which obviously impacts postoperative mortality. Clearly, all these conditions can be targeted peri-operatively, e.g., applying an intensified antibiotic perioperative treatment in those who are more prone to acquire pneumonia, or a more thorough cardiac work-up on those who are likely to develop postoperative arrhythmias. Each of these conditions could be assigned a specific scoring system with which the individual risk can be predicted.

In their study in the European Fournal of Cardiothoracic Surgery, Hino and colleagues make an important step towards the right direction by identifying a subgroup of patients older than 80 years that qualifies best for operation or for which an operation is rather considered to be harmful. They included 337 patients from 7 institutions and analyzed those for risk factors for postoperative complications and long-term survival (13). One of the two main findings of Hino and colleagues was that male gender was an independent risk factor for postoperative complications. This finding is supported by Saji and colleagues who identified male gender to be an independent risk factor for postoperative complications when designing a risk scoring system (11) and also by a study from Stamenovic and colleagues analyzing 45 octogenarians (14). Hino listed 17 different complications in their study, however, it remains open by which of those complications the male patients were affected by. If this would be known, some of the complications might be prevented by a specific preoperative treatment or by excluding those patients from the operation. As the second main finding, Hino and colleagues identified operation time to be a risk factor for postoperative complications. This factor was also reported to be relevant before by others (15). The invention of minimally invasive thoracoscopic and robotic resection treatment modalities with less operation time clearly provides advantages for the patient. However, centers that are not well experienced and trained in thoracoscopic surgery, the procedure will be more time-consuming which counteracts the beneficial aspects of minimally invasive surgery.

Surprisingly, Hino reported that pulmonary fistula is the most common postoperative complication in their cohort. This complication is well known to be responsible for a higher morbidity and mortality. As authors applied partial resection as the second most often procedure employed in their patient cohort, the reported high rate of fistula is largely unexplained, unless there was severe emphysema in those patients. The conclusion than would be to avoid surgery on such patients.

Considering the poor outcome with an estimated 5-year survival of only $7 \%$ in patients older than 80 years with stage I non-small cell lung carcinoma if they do not receive any form of treatment $(8,16)$, standard therapy in lung cancer treatment in these patients seems mandatory. This includes the systematic dissection of lymph nodes (17-19). In the light of a longer operation, Hino and colleagues suggest to reconsider the indication of lymph node resection in octogenarians. Indeed, Okami and colleagues showed in a cohort of 367 patients that mediastinal lymph node dissection increased the risk of postoperative complications (20). Also, Ogawa found that lymph node dissection increases the incidence of postoperative complications (21). It is not entirely clear from those studies if the higher incidence of postoperative complications was related to a prolonged operation time or from the procedure of lymph node dissection itself. In well trained and practiced thoracic surgical hands, lymph node dissection does not significantly prolong the total operation time of a procedure nor, if properly done, it should harm the patient, so it remains questionable if this step of the operation impacts postoperative outcome.

For a better risk stratification for postoperative mortality and morbidity as well as for postoperative survival, Hino and colleagues employed the Charlson Comorbidity Index (CCI), a scoring system for a range of comorbid systemic diseases which aligns with the general condition of the patient (22), and the Glasgow prognostic score (GPS), a score calculated from levels of serum albumin and C-reactive protein, which is representative of the systematic inflammatory status (23). For their patient cohort, authors could convincingly show the CCI and GPS to be of predictive reliability both being in accordance with previous studies. The 5 -year survival benefit of females (79\%) vs. males (57.7\%) was impressive to see (13). Authors thus made a major effort to narrow down the group of octogenarians who are recommended to operate on. The Japanese population is fortunate to enjoy a long and 
relatively healthy life with less comorbidities as authors report of $40 \%$ octogenarian patients with no comorbidities.

However, both of them, neither the CCI nor the GPS consider the very individual risk of a patient. Every single patient carries a unique individual risk pattern along with its individual susceptibility for developing intraand postoperative complications. This risk pattern and susceptibility could be specifically cared for by an individual and differentiated diagnostic and therapeutic work-up prior to the procedure. In that context, past and present everyday physical and mental activities as well as life style needs to be taken into account to detect the risk pattern of an elderly patient. On that base, it will be better possible to "predict" on which patient an operation can be recommended and on which patient the operation should be discouraged. We live today in an era of personalized medicine when it comes to e.g., targeting a patient's individual expression of surface markers such as PD-L1 by checkpoint inhibitors. Similarly, we need to analyze the individual risk pattern of an octogenarian which should be the new definition of "selection". This will be the most important task of future studies towards an appropriate surgical treatment of each octogenarian and should provide the clinician with a guideline on which patients safely operate on.

\section{Acknowledgements}

None.

\section{Footnote}

Conflicts of Interest: The author has no conflicts of interest to declare.

\section{References}

1. Korc-Grodzicki B, Downey RJ, Shahrokni A, et al. Surgical considerations in older adults with cancer. J Clin Oncol 2014;32:2647-53.

2. Kiernan PD, Khandhar SJ, Fortes DL, et al. Thoracic surgery in octogenarians: CVTSA/Inova Fairfax hospital experience, 1990 to 2009. Am Surg 2011;77:675-80.

3. Tutic-Horn M, Gambazzi F, Rocco G, et al. Curative resection for lung cancer in octogenarians is justified. $\mathrm{J}$ Thorac Dis 2017;9:296-302.

4. Calado T, Antunes M, Cabral D, et al. Surgical Treatment for Elderly Patients with Lung Cancer. Rev Port Cir Cardiotorac Vasc 2017;24:144.
5. Ganti AK, Shostrom V, Alorabi M, et al. Early Stage NonSmall-Cell Lung Cancer in Octogenarian and Older Patients: A SEER Database Analysis. Clin Lung Cancer 2016;17:285-91.

6. Berry MF, Onaitis MW, Tong BC, et al. A model for morbidity after lung resection in octogenarians. Eur J Cardiothorac Surg 2011;39:989-94.

7. Brunelli A, Kim AW, Berger KI, et al. Physiologic evaluation of the patient with lung cancer being considered for resectional surgery: Diagnosis and management of lung cancer, 3rd ed: American College of Chest Physicians evidence-based clinical practice guidelines. Chest 2013;143:e166S-90S.

8. Raz DJ, Zell JA, Ou SH, et al. Natural history of stage I non-small cell lung cancer: implications for early detection. Chest 2007;132:193-9.

9. Vazirani J, Moraes J, Barnett S, et al. Outcomes following resection of non-small cell lung cancer in octogenarians. ANZ J Surg 2018;88:1322-7.

10. Powell HA, Tata LJ, Baldwin DR, et al. Early mortality after surgical resection for lung cancer: an analysis of the English National Lung cancer audit. Thorax 2013;68:826-34.

11. Saji H, Ueno T, Nakamura H, et al. A proposal for a comprehensive risk scoring system for predicting postoperative complications in octogenarian patients with medically operable lung cancer: JACS1303. Eur J Cardiothorac Surg 2018;53:835-41.

12. Detillon DD, Veen EJ. Postoperative Outcome After Pulmonary Surgery for Non-Small Cell Lung Cancer in Elderly Patients. Ann Thorac Surg 2018;105:287-93.

13. Hino H, Karasaki T, Yoshida Y, et al. Risk factors for postoperative complications and long-term survival in lung cancer patients older than 80 years. Eur J Cardiothorac Surg 2018;53:980-6.

14. Stamenovic D, Messerschmidt A, Schneider T. Surgery for lung tumors in the elderly: A retrospective cohort study on the influence of advanced age (over 80 years) on the development of complications by using a multivariate risk model. Int J Surg 2018;52:141-8.

15. Shiono S, Abiko M, Sato T. Postoperative complications in elderly patients after lung cancer surgery. Interact Cardiovasc Thorac Surg 2013;16:819-23.

16. Varlotto JM, Decamp MM, Flickinger JC, et al. Would screening for lung cancer benefit 75- to 84-year-old residents of the United States? Front Oncol 2014;4:37.

17. Shen-Tu Y, Mao F, Pan Y, et al. Lymph node dissection and survival in patients with early stage nonsmall cell lung 
cancer: A 10-year cohort study. Medicine (Baltimore) 2017;96:e8356.

18. Krantz SB, Lutfi W, Kuchta K, et al. Improved Lymph Node Staging in Early-Stage Lung Cancer in the National Cancer Database. Ann Thorac Surg 2017;104:1805-14.

19. Mueller MR. Tailored management of stage IIIa nonsmall-cell lung cancer in the era of the 8th edition of the TNM classification for lung cancer. Future Oncol 2018;14:5-11.

20. Okami J, Higashiyama M, Asamura H, et al. Pulmonary resection in patients aged 80 years or over with clinical stage I non-small cell lung cancer: prognostic factors for overall survival and risk factors for postoperative

Cite this article as: Jungraithmayr W. Lung cancer surgery in octogenarians revisited-risk factors and survival in a long lifespan population. J Thorac Dis 2018;10(12):6423-6426. doi: $10.21037 /$ jtd.2018.12.31 complications. J Thorac Oncol 2009;4:1247-53.

21. Ogawa F, Wang G, Matsui Y, et al. Risk factors for postoperative complications in the elderly with lung cancer. Asian Cardiovasc Thorac Ann 2013;21:313-8.

22. Charlson ME, Pompei P, Ales KL, et al. A new method of classifying prognostic comorbidity in longitudinal studies: development and validation. J Chronic Dis 1987;40:373-83.

23. McMillan DC, Forrest LM, O'Gorman P, et al. Performance status of male and female advanced cancer patients is independently predicted by mid-upper arm circumference measurement. Nutr Cancer 2002;42:191-3. 